\title{
REGULAÇÃO EM SAÚDE: APLICABILIDADE PARACONCRETIZAÇÃO DO PACTO DE GESTÃO DO SUS
}

\author{
Adail Afrânio Marcelino do Nascimento¹, Ana Kelve Damasceno², Maria Josefina da Silva³, Maria Veronica Sales da
} Silva ${ }^{4}$, Aline Rodrigues Feitoza ${ }^{5}$

\begin{abstract}
RESUMO: O objetivo da pesquisa é analisar o conceito de regulação em saúde, descrito na Política Nacional de Regulação, para a concretização do Pacto pela Saúde 2006. A coleta de dados teve por base os conceitos que procederam as informações colhidas de revistas indexadas, livros e portal do Ministério da Saúde, complementada por busca no banco de dados da BIREME, referente ao período de 2001 a 2006. Foram encontrados 19 artigos com os descritores regulação, saúde e usuário. Observou-se que o processo de regulação ainda é incipiente e torna-se necessário o enfrentamento de temas mais complexos e estruturantes, como o desafio de entender a natureza dessa regulação, seus avanços e limites, a dimensão da organização do subsetor, o financiamento da oferta de serviços, as modalidades assistenciais, suas redes e a complexidade dessas relações.
\end{abstract}

PALAVRAS-CHAVE: Regulação; Saúde; Usuário.

\section{HEALTH REGULATION: APPLICABILITY TO THE CONCRETION OF THE UNIFIED HEALTH SYSTEM MANGEMENT PACT}

\begin{abstract}
The objective of this study is to analyze the concept of regulation in health described in the National Politics in Regulation, for the concretion of the Pact for Health 2006. The collection of data was based on the descriptors that led to the information retrieval from indexed magazines, books and the Ministry of Health internet portal. The research was also carried from the BIREME database, referring to the period from 2001 to 2006. Nineteen articles were found, with the descriptors: regulation, health and user. We observe that the regulation process is still incipient. It is necessary the confrontation of more complex and structuring subjects such as the challenge of understanding the nature of this regulation, its advances and limits, the dimension of the organization of the subsector, the financing of services, the assistance modalities, its networks, and the complexity of these relations.
\end{abstract}

KEYWORDS: Regulation; Healtn; User.

\section{REGULACIÓN EN SALUD: APLICABILIDAD PARA CONCRETIZACIÓN DEL PACTO DE GESTIÓN DEL SISTEMA ÚNICO DE SALUD (SUS)}

RESUMEN: El objetivo de la investigación es analizar el concepto de regulación en salud, descripto en la Política Nacional de Regulación, para la concretización del Pacto por la Salud 2006. La colecta de datos tuvo por base los conceptos que procedieron las informaciones recogidas de revistas indexadas, libros y portal del Ministerio de la Salud, complementada por búsqueda en el banco de datos de la BIREME, referente al periodo de 2001 a 2006. Fueron encontrados 19 artículos con los descriptores Regulación, Salud y Usuario. Se observó que el proceso de regulación todavía es incipiente y se vuelve necesario el enfrentamiento de temas más complejos y estructurados, como el desafío de comprender la naturaleza de esa regulación, sus avanzos y límites, la dimensión de la organización del subsector, el financiamiento de la oferta de servicios, las modalidades asistenciales, sus redes y la complejidad de esas relaciones.

PALABRAS CLAVE: Regulación; Salud; Usuario.

${ }^{1}$ Mestre. Professor do Curso de Enfermagem da Faculdade Integrada da Grande Fortaleza-FGF-CE.

${ }^{2}$ Doutora. Professora do Curso de Enfermagem da Universidade Federal do Ceará-UFC.

${ }^{3}$ Doutora. Professora do Curso de Enfermagem da UFC.

${ }^{4}$ Doutora. Professora da Pós-Graduação em Auditoria em Saúde da Ratio Faculdade de Fortaleza.

${ }^{5}$ Doutora. Professora do Curso de Enfermagem da Universidade de Fortaleza-UNIFOR-CE.

Autor correspondente:

Adail Afrânio Marcelino do Nascimento

Rua Dr. Hélio Viana, 1594 - 60320-240 - Fortaleza-CE

Recebido: 12/12/08

E-mail: afranio@fgf.edu.br

Aprovado: 20/06/09

Cogitare Enferm 2009 Abr/Jun; 14(2):346-52 


\section{INTRODUÇÃO}

Para avançar na consolidação do Sistema Único de Saúde-SUS é absolutamente necessário possibilitar que a formulação da política para o setor saúde ocorra mediante espaços, que concretizem a aproximação entre construção da gestão descentralizada do SUS, o desenvolvimento da atenção integral como acolhida e responsabilidade do conjunto integrado do sistema de saúde e o fortalecimento da participação popular, com poder deliberativo.

A descentralização dos serviços de saúde teve impacto na melhoria dos níveis de saúde da população, havendo fortalecimento na percepção da necessidade de regionalizar e hierarquizar os serviços, para assegurar a assistência integral, resolutiva e com qualidade.

Mesmo com a descentralização da saúde, não foi possível garantir a regulação do acesso à assistência, na qual reflete ações consubstanciadas no modelo anterior de saúde, ou seja, alto grau de centralização, verticalização das ações, das decisões e normatizações, caracterizando fragmentação da ação do Estado, no aspecto de promover a saúde da população.

No entanto, observou-se que os Estados e Municípios não estão desenvolvendo métodos de sistemas de planejamento eficazes, ou seja, não se adequaram aos moldes da Norma Operacional de Assistência em Saúde-NOAS 01/2002, no que diz respeito ao controle, avaliação, regulação e auditoria. E os Estados e Municípios que incorporaram a modalidade não estão exercitando o controle, avaliação, regulação e auditoria ${ }^{(1)}$.

Atualmente, no Pacto de Gestão do SUS, os gestores pactuam responsabilidades entre os entes federados, no campo da gestão do Sistema e da atenção à saúde. Esse pacto firmado entre os gestores do SUS, a partir de uma unidade de princípios que, guardando coerência com a diversidade operativa, respeita as diferenças locorregionais, agrega os pactos anteriormente existentes, reforça a organização das regiões sanitárias instituindo mecanismos de cogestão e planejamento regional, fortalece os espaços e mecanismos de controle social, qualifica o acesso da população à atenção integral à saúde, redefine os instrumentos de regulação, programação e avaliação, valoriza a macrofunção de cooperação técnica entre os gestores e propõe um financiamento tripartite, que estimula critérios de equidade nas transferências fundo a fundo ${ }^{(2)}$.

Portanto, observou-se que o processo de regulação ainda é incipiente e torna-se necessário o enfrentamento de temas mais complexos e estruturantes, como o desafio de entender a natureza dessa regulação, seus avanços e limites, a dimensão da organização do subsetor, o financiamento da oferta de serviços, as modalidades assistenciais, suas redes e a complexidade dessas relações. A compreensão do modelo assistencial praticado só se faz na medida em que se entende o processo de regulação existente ${ }^{(3)}$.

Após vinte anos da construção do SUS, a União, Estados e Municípios definiram novas responsabilidades em relação à saúde do País. Foram realizados vários encontros dos gestores do SUS, para assinar o Pacto pela Saúde 2006, na reunião da Comissão Intergestores Tripartite- CIT, aprovado pelo Conselho Nacional de Saúde-CNS, em fevereiro de 2006. Esse pacto aborda três dimensões: o Pacto pela Vida, o Pacto em Defesa do SUS e o Pacto de Gestão, publicado na Portaria GM/MS n. 399, de fevereiro de 2006, sendo regulamentado pela Portaria GM/MS n. 699, de março de $2006^{(1)}$.

O Pacto pela Saúde 2006 institui novas bases para que o SUS alcance processo mais solidário de gestão, é traduzido como um termo de compromisso entre entes públicos, no qual se definem responsabilidades sanitárias e de gestão, de resultados a serem alcançados, possibilitando sua avaliação e controle público. Sua efetividade se dá por meio da adesão do gestor com aprovação do Conselho de Saúde ${ }^{(2)}$.

Esse pacto traz uma mudança importante para a gestão do SUS, haja vista que viabiliza a incorporação de ações estratégicas que irão subsidiar a implementação da gestão na área de planejamento, controle, avaliação, regulação e auditoria, sendo fundamental que os gestores, profissionais de saúde, conselheiros, prestadores e outros atores envolvidos conheçam e compreendam as mudanças propostas na organização do SUS.

A dimensão que se destaca no estudo é o Pacto de Gestão, com as ações de regulação em saúde devem ser efetivadas, em âmbito nacional, com um posicionamento político de fortalecer as diretrizes do SUS, sendo necessário um processo de conscientização dos gestores acerca da organização desse setor. Ainda, que essas ações devem reforçar e qualificar as funções de gestão, otimizando os recursos de custeio da assistência, qualificando o acesso e, consequentemente, proporcionando aos usuários do SUS um acesso à saúde equânime, integral e de qualidade ${ }^{(2)}$. 
Nesse contexto de mudanças, para definir a regulação em saúde e seus objetivos na gestão de sistemas de serviços de saúde, é importante compreender a diferença da regulação em geral e regulação estatal no setor saúde, buscando discriminar melhor suas atividades, seus objetos, assim como as competências dessa função, para cada esfera de gestão, no âmbito do SUS.

Os conceitos, as práticas e finalidades da regulação em saúde surgiram a partir de 2002, quando o Ministério da Saúde do Brasil iniciou o debate sobre os conceitos e as práticas da regulação que, tais como as de controle, avaliação e auditoria, não têm sido uniformes.

Refletindo sobre a perspectiva do fortalecimento da capacidade gestora dos Estados e Municípios, na organização das redes de serviços de saúde e no planejamento das ações, verificou-se que a regulação em saúde não ocorre de forma eficaz ou contextualizada, levando-as ao não cumprimento das referências pactuadas e, consequentemente, aos obstáculos como as barreiras físicas e burocráticas do acesso da população às ações e serviços.

Contudo, a realidade da construção do SUS evidenciou a necessidade de propor novas formas de lidar com a fragmentação da gestão pública, surgindo novas propostas de programas e projetos para a saúde. A proposta deste estudo justifica-se, pois, pelo surgimento de novos conceitos e legislação do SUS, acerca da regulação assistencial, sendo um desafio para gestores, profissionais de saúde e usuários a concretização e efetivação desse pacto.

Assim, o pacto de gestão do SUS define a Regulação como diretriz que configura uma linha de financiamento de custeio específica, através do bloco de financiamento da Gestão; conceitua as principais estruturas regulatórias; lista os princípios orientadores do processo de Regulação; define meta e explicita as responsabilidades dos Gestores Municipais, Estaduais, do Distrito Federal e do Gestor Federal ${ }^{(1)}$. Tendo em vista a relevância da regulação em saúde para a organização da oferta dos serviços de saúde, propôsse a analisar o conceito de regulação em saúde descrito na Política Nacional em Regulação na concretização do Pacto de Gestão do SUS.

\section{METODOLOGIA}

Trata-se de estudo documental reflexivo, que buscou analisar os conceitos de regulação em saúde na concepção do Pacto de Gestão do SUS.

O estudo foi realizado mediante busca sistemática na LILACS-Literatura Latino-Americana e do Caribe em Ciências de Saúde. Essa é uma base de dados cooperativa da Rede BVS, que compreende a literatura relativa às ciências da saúde publicada nos países da América Latina e Caribe.

Foram utilizados como critério de seleção os descritores em saúde: Regulação; Saúde; Usuário. Quanto ao período para captação dos dados foi delimitada entre os anos de 2001 a 2006. A coleta de dados teve por base o estudo de artigos, livros e portarias ministeriais, sendo observadas 25 referências, das quais 19 atenderam ao objetivo proposto.

Durante a realização da captação, deparou-se com uma escassez de estudos ou arquivos relacionados ao tema em foco. Identificaram-se trabalhos científicos que abordavam a regulação estatal, falhas de mercado e falhas do Estado, governanças aplicada na regulação, tomada decisória, marcos conceituais sobre a regulação e outros.

A análise dos documentos, integralmente lidos, interpretados e agrupados, originou duas categorias: conceito de regulação e a aplicação do conceito de regulação na política nacional de regulação.

\section{ANÁLISE E DISCUSSÃO}

\section{Conceituando a regulação em saúde}

Existem conceitos que definem a regulação estatal na saúde apenas como o ato de regulamentar, de elaborar as regras. A regulação em saúde é um conjunto de ações mediatas, que se interpõem entre as demandas dos usuários e seu acesso aos serviços de saúde, traduzidas em fluxos, protocolos assistenciais, centrais de leitos, centrais de consultas e exames, além dos processos de trabalhos correspondentes. A concepção ampliada de regulação no setor saúde, além da fiscalização e controle, incorpora funções de regulação à condução política, à análise da situação, ao planejamento e à comunicação, confundindo-se, assim, com o conceito de gestão ${ }^{(4)}$.

O termo Regulação, aplicado ao setor saúde no país, tem diversos entendimentos, concepções e práticas, sendo definida como regulação da atenção à saúde, a regulação assistencial e a regulação do acesso. Dessa forma, a regulação é definida em três dimensões: regulação da atenção à saúde, que "compreende a contratação, a regulação assistencial, o controle 
assistencial, a avaliação da atenção à saúde, a auditoria assistencial e as regulamentações da vigilância epidemiológica e sanitária”; regulação assistencial, definida como o "conjunto de relações, saberes, tecnologias e ações, que intermedeiam a demanda dos usuários por serviços de saúde e o acesso a eles”; e regulação do acesso, considerada como o "estabelecimento de meios e ações para a garantia do direito constitucional de acesso universal, integral e equânime"(1).

A função de regulação é entendida como estratégia em estreita interface com o planejamento, o controle, a avaliação e com os diversos níveis de complexidade da assistência, buscando garantir a assistência integral aos usuários do SUS. A regulação é um dos instrumentos de gestão que viabiliza o ordenamento da relação dos usuários com os prestadores de saúde ${ }^{(4-5)}$.

Assim, os marcos conceituais da regulação em saúde delimitam claramente a regulação de acesso dos usuários aos serviços assistenciais, trazendo também a noção de complexos reguladores. Desse modo, a Política de Nacional de Regulação, no sistema de serviços de saúde, vem trazendo ações ou instrumentos de operacionalização, através do pacto de gestão do SUS, a efetivação dos princípios e diretrizes do SUS, viabilizando o acesso equânime e oportuno à atenção integral e de qualidade, a universalidade e a garantia de direitos sociais dos usuários.

Na busca do conceito de Regulação em trabalhos, portarias ministeriais, artigos e livros, encontrou-se a expressão Regulação como ações diretas e finais de atenção à saúde. No aspecto econômico, a Regulação é entendida como a capacidade de intervenção estatal nos mercados. Num conceito mais restrito, pode ser considerada como a compatibilização de oferta e serviços assistenciais ${ }^{(6)}$. A palavra regulação, neste sentido, traz raízes fincadas na intervenção do Estado, destacando a regulamentação e a fiscalização de algo. No decorrer dos anos, sempre o Estado é soberano nas decisões, fato que vem desde o ínicio da história das políticas de saúde, como, por exemplo, no Brasil.

O Pacto Pela Saúde define regulação como um conjunto de relações, saberes, tecnologias e ações que intermediam a demanda dos usuários por serviços de saúde e o acesso a eles. A Regulação do acesso é o estabelecimento de meios e ações para a garantia do direito constitucional de acesso universal, integral e equânime, independente de pactuação prévia estabelecida na Programação Pactuada e IntegradaPPI ou da disponibilidade de recursos financeiros ${ }^{(3)}$.

Portanto, a regulação da assistência à saúde é uma estratégia de serviço adequada à necessidade do usuário, de forma equânime, ordenada, oportuna e qualificada, que deverá ser efetivada através de complexos reguladores, que façam congregar unidades de trabalho responsáveis pela regulação das urgências, consultas, leitos, entre outros. Desse modo, a regulação é definida como um conjunto de ações e instrumentos para organizar a oferta, conforme a necessidade da população, estabelecendo competências, fluxos e responsabilidades, a fim de garantir o acesso com qualidade e resolubilidade, em todos os níveis de atenção à saúde ${ }^{(7)}$.

Após análise dos conceitos de regulação, especialmente em saúde, faz-se necessário compreender a organização e a hierarquização do SUS, tendo como pressuposto básico a regionalização, considerada como princípio orientador, constitucionalmente definido, que integra as ações e serviços de saúde numa rede regionalizada e hierarquizada.

Assim, para conseguir a regulação em saúde é preciso entender o que é um processo técnico, científico e político de intervenção no sistema de saúde e de coordenação da integração dos sistemas municipais, a fim de adequar a relação entre a oferta e a demanda das ações e serviços de saúde e obter resultados superiores, do ponto de vista social, viabilizando o acesso à saúde.

Na perspectiva da concretização das diretrizes da regulação no Pacto de Gestão do SUS, onde estabeleceram que cada prestador responde apenas a um gestor; a regulação dos prestadores de serviços deve ser, preferencialmente, do Município, conforme desenhado na rede de assistência pactuada na Comissão Intergestores Bipartite-CIB, observando o termo de compromisso de gestão do pacto; a regulação das referências intermunicipais é responsabilidade do gestor estadual $^{(3)}$.

Resumidamente, a regulação dos prestadores não tem um modelo único para o País, pois cada Comisssão Intergestores Bipartite-CIB poderá adotar o modelo que melhor se adapte à realidade do Estado e dos Municípios envolvidos. No entanto, a regulação assistencial não é, exclusivamente, prerrogativa de uma esfera do governo.

\section{Aplicação do conceito de regulação na política nacional de regulação}


Inicialmente, a regulação em saúde deve ser pensada sempre no contexto dos princípios norteadores do SUS, e não apenas como forma de racionalizar os recursos existentes. A Política Nacional de Regulação, no tocante à função de regulação, propõe três eixos a serem operacionalizados pelos gestores, conforme estabelecido nas diretrizes do Pacto de Gestão do SUS, os quais são definidos como os Recursos financeiros para a implantação e para o custeio dos Complexos Reguladores, os instrumentos para operacionalização dos Complexos Reguladores e o Programa de capacitação permanente de recursos humanos.

O primeiro eixo traz o financiamento inicial, visando à implantação dos complexos reguladores e contempla uma estrutura mínima composta por: estrutura logística, elétrica e de telefonia, inerentes ao seu funcionamento; infra-estrutura de mobiliário e equipamentos, inclusive de informática, o aplicativo do Sistema de Regulação-SISREG III. Em relação ao eixo dois são definidos instrumentos para operacionalização dos Complexos Reguladores como o pacto de gestão, que define toda a política norteadora do SUS; os contratos com os prestadores de serviços, que expressam formalmente a oferta de serviços de saúde e sua coerência com as necessidades populacionais; o cadastramento das pessoas através do Cartão Nacional de Saúde-CNS e do Cadastro Nacional de Estabelecimentos e Profissionais-CNES; o Plano Diretor de Regionalização-PDR; a Programação Pactuada e Integrada-PPI; o Programa Nacional de Avaliação de Serviços de Saúde-PNASS e outros.

O eixo três aborda o Programa de capacitação permanente de recursos humanos. A capacitação pretende formar multiplicadores proporcionando à Gestão Estadual e as Gestões Municipais as condições para o estabelecimento de um pólo de educação permanente, que garanta a formação de novos atores no processo de regulação, bem como a contínua atualização $0^{(8)}$.

Com relação aos complexos reguladores definidos no primeiro eixo, esses deverão estruturar a relação entre os vários serviços, formando uma rede de atenção, determinando o fluxo dos pacientes no sistema e gerando um canal de comunicação aberto à população, através dos quais os pedidos são recebidos, avaliados e hierarquizados e a resposta fornecida, de acordo com as necessidades de cada caso, com o consequente acompanhamento da solução( ${ }^{(9)}$.

Entende-se que os Complexos Reguladores são estruturas que agregam todas as ações da Regulação do Acesso à Assistência, de modo articulado e integrado, adequando a oferta de serviços de saúde à demanda, que mais se aproxima das necessidades reais em saúde.

Essas estruturas permitem aos gestores articular e integrar os dispositivos de Regulação do Acesso, como as Centrais de Internação, as Centrais de Marcação de Consultas e Exames Especializados, os Protocolos Assistenciais com outras ações de Regulação da Atenção em Saúde como: Contratação, Controle e Avaliação, assim como em outras funções de gestão, como programação e regionalização. Regular a oferta e a demanda por meio de Complexos Reguladores possibilitam a organização das ações de regulação do acesso, no sentido de garantir a integralidade das ações de Atenção à Saúde, com qualidade e equidade ${ }^{(8)}$.

De fato, a efetivação dessa política depende da existência da rede assistencial estruturada e com o fluxo estabelecido, utilizando instrumentos de acompanhamento e monitoramento de conformidades, na questão da operacionalização, ou seja, instituído o complexo regulador.

Observa-se, em alguns municípios brasileiros, que a implantação das Centrais de Marcação de Consultas-CMC tem funcionado como uma estratégia necessária para operacionalizar o Sistema de Referência e Contra-referência, a partir de um modelo assistencial que propõe a reorganização do acesso dentro do Sistema de Saúde. As CMC funcionam como um centro aglutinador das consultas especializadas. A função das CMC é, portanto, contribuir para a regulação das ações de saúde ao administrar as consultas especializadas, geradas pelas unidades básicas de saúde nos Municípios ${ }^{(10)}$.

Portanto, considera-se que a Central de Regulação é uma ferramenta de gestão, constituindose em um instrumento que fortalece o papel do gestor, dando maior transparência à relação com a rede prestadora, tornando possível planejar e distribuir equitativamente os recursos de saúde de uma região, tendo como referência sua respectiva Programação Pactuada Integrada-PPI.

A Central de Regulação é uma estrutura que compreende toda a ação meio do processo regulatório, ou seja, é o local que recebe as solicitações de atendimento, avalia, processa e agenda, garantindo o atendimento integral de forma ágil e de qualidade aos usuários do Sistema de Saúde. Faz-se necessário o conhecimento da capacidade instalada nas unidades 
prestadoras de serviços e a oferta dessas unidades, pois isso possibilitará a identificação dos déficits e falhas do sistema e a tomada de decisões de forma consistente, eficaz e eficiente em cada esfera do governo $^{(9)}$.

Entende-se que a função de regulação possui uma estreita relação com o planejamento, o controle, a avaliação e com os diversos níveis de complexidade da assistência, buscando garantir a assistência integral aos usuários do SUS.

A efetividade da política nacional de regulação as Centrais de Regulação, no tocante à implantação do complexo regulador, proporciona ao Gestor a possibilidade de avaliar, a qualquer momento, todos os procedimentos realizados no paciente pesquisado, chegando ao seu histórico clínico, com possibilidade de acompanhar todos os resultados de avaliações e exames realizados por ele.

No Estado do Ceará, a implantação do complexo regulador ocorreu através da criação de 22 microrregiões de saúde, as quais possuem uma Central de Regulação informatizada e organizada, conforme o fluxo dos pacientes na região. A regionalização adotada foi pactuada com os Gestores Municipais e Estaduais, sendo utilizada tecnologia da informação na gestão da saúde, implantando a Rede de Centrais de Regulação para internações e exames de alta e média complexidade, direcionados para todas as regiões do Estado(11).

A Central de Regulação Estadual do SUS no Ceará-CRESUS-CE recebe e direciona, em tempo real, toda a demanda de referências intermunicipais de consultas especializadas e exames, internações hospitalares eletivas e de urgência e emergência. A utilização da CRESUS, com o processo de implantação concluído em 2004, vem aumentando tanto em agendamento ambulatorial como nas transferências hospitalares. Em janeiro de 2004, foram 19.723 agendamentos de consultas e, em 2005, aumentou para 49.787 agendamentos. Em 2004, foram 8.178 transferências, passando para 18.316 em $2005^{(11)}$.

A Central verifica a situação dos leitos da região em termos de ocupação/especialidades e perfil tecnológico associado, poupando o usuário da peregrinação por diferentes Municípios e hospitais, na busca de internação e de consultas eletivas em especialidades não ofertadas nos Municípios.

A CRESUS-CE vem desenvolvendo bom desempenho na melhoria de acesso como: a interferência proativa nos fluxos assistenciais; a utilização de protocolos de acesso e mediação (regulação) médica para avaliação de cada demanda assistencial, permitindo que os Gestores Municipais e Estaduais aplicassem os controles estabelecidos pela normatização vigente do SUS ${ }^{(11)}$.

No entanto, é importante esclarecer que todos esses benefícios são em maior ou menor escala, parciais, ou seja, ainda estão em construção. A CRESUS encontra-se em operação desde 2002, mas sua implantação total no Estado só foi alcançada em 2004, o que a torna um instrumento com apenas dois anos de uso efetivo em toda a sua abrangência.

No entanto, a CRESUS, notadamente, a regulação deve ser pensada sempre no contexto dos princípios norteadores do SUS e não apenas como forma de racionalizar os recursos existentes. Deve contribuir com o fortalecimento da gestão, na garantia do direito à saúde, à universalidade e integralidade, com eficiência, eficácia e efetividade. O pacto reforça no SUS o movimento de uma gestão pública baseada em resultados, que só podem ser obtidos de forma satisfatória, a partir de um planejamento e de sua implementação de forma eficiente ${ }^{(12)}$.

\section{CONSIDERAÇÕES FINAIS}

Entende-se que a Regulação em saúde é uma necessidade absoluta no Sistema Único de Saúde-SUS, através da qual são operadas as pactuações entre Municípios e Regiões da unidade federada.

A partir da análise dos conceitos estudados, percebe-se que há várias atribuições e competências acerca da regulação, os quais são abrangentes e respondem a uma necessidade de regulação num sentido amplo, não somente no que se refere à fiscalização e controle, mas, também, ao estabelecimento de regras e mecanismos para a orientação do sistema. Esses conceitos buscam articular e integrar as ações de regulação, controle, avaliação e auditoria, em que as ações de regulamentação, fiscalização, controle, avaliação e auditoria da produção de bens e serviços em saúde sirvam, de fato, como ações mediatas, oportunas e facilitadoras das ações fins da atenção à saúde, efetivem a universalidade e equidade do acesso, a resolubilidade e integralidade do cuidado, bem como as ações meio que ajudem a criar as condições para uma atenção à saúde, pautada pelas demandas, necessidades e direitos de usuários cidadãos

Na linha do fortalecimento e da concretização, o 
Pacto de Gestão do SUS traz importantes mudanças para a reorganização dos processos de gestão e de regulação do sistema, destacando o papel fundamental dos entes federados, Municípios, Estados e Ministério da Saúde, para melhorar e qualificar o acesso do cidadão aos serviços de saúde, na busca da universalidade e da integridade, com eficiência, eficácia e efetividade.

\section{REFERÊNCIAS}

1. Ministério da Saúde (BR). Conselho Nacional de Secretários de Saúde. Regulação em saúde. Brasília, DF: CONASS; 2007. (Coleção Progestores).

2. Ministério da Saúde (BR). Secretaria Executiva. Regulamento dos pactos pela vida e de gestão. Brasília, DF; 2006.

3. Mesquita MAF. A regulamentação da assistência da saúde suplementar: legislação e contexto institucional. Rio de Janeiro: Ministério da Saúde; 2002.

4. Ministério da Saúde (BR). Secretaria da Assistência à Saúde. Regulação no setor de saúde: em direção aos seus fundamentos públicos. Brasília, DF; 2004.

5. Ministério da Saúde (BR). Controle, regulação e avaliação. Brasília, DF; 2002.

6. Batista S. Regulação da assistência. Brasília, DF; 2000. Digitado.

7. Ministério da Saúde(BR). Portaria n. 1.571, de 29 de junho de 2007. Estabelece incentivo financeiro para implantação e/ou implementação de Complexos Reguladores. Diário Oficial da União. 24 jul 2007; Seção 1.

8. Locks MTR. Central de Marcação de Consultas da Grande Florianópolis: um estudo de caso [dissertação]. Florianópolis (SC): Universidade Federal de Santa Catarina; 2002.

9. Secretaria de Saúde do Estado do Ceará. A saúde no Ceará: uma construção de todos: relatório de gestão da Secretaria de Saúde do Estado do Ceará. Fortaleza: OMNI; 2006.

10. Silva MV. Avaliação do Complexo Regulador da segunda Microrregional de Saúde-CE [tese]. Fortaleza (CE): Universidade Federal do Ceará; 2008.

11. Fortier CC. Contribuições da CRESUS no acesso aos serviços públicos de saúde no Estado do Ceará [monografia]. Fortaleza (CE): Universidade Federal Ceará; 2006.
12. Ribeiro LC, Ribeiro M, Dias KS. O diagnóstico administrativo e situacional como instrumento para o planejamento de ações na estratégia saúde da família. Cogitare Enferm. 2008 Jul/Set;13(3):448-52. 